\title{
Dynamic Resistance of Multi-Layered Protective Elements Under Impact Loads
}

\author{
Leopold Kruszka ${ }^{1, *}$, Iurii Vorobiov ${ }^{2}$, Nataliia Ovcharova ${ }^{2}$ \\ ${ }^{1}$ Military University of Technology, Civil Engineering and Geodesy Faculty, 00-908 Warsaw, Poland \\ ${ }^{2}$ A.N. Podgorny Institute of Mechanical Engineering Problems NAS, Kharkov, 61046, Ukraine
}

\begin{abstract}
To ensure the dynamic strength of modern constructions, protective elements are used. Studies have shown that even with the use of double-layer elements from similar materials, multilayer elements have enhanced protective properties with a smaller overall thickness compared to single-layer elements. Even greater effect is achieved when using layers of different materials. These features are widely used in the creation of linings to strengthen the bodies of gas turbine engines when exposed to fragments of blades and foreign objects. Numerical studies of the stress-strain state of three-layer element from two thin layers of a titanium alloy and an average ceramic layer under the influence of projectile with different velocities are carried out. The top layer of the titanium alloy perceives the main local load and plastic deformations occur up to the formation of the crater. In the average ceramic layer, the extensive deformations develop, during which the basic energy of impact is absorbed. The third layer limits the velocity of deformation and increases the protective properties of the element. Thus, it is shown that the multilayer elements can have improved protective properties with a general reduction of the weight of structure.
\end{abstract}

\section{Introduction and statement of the problem}

Modern constructions often work under the impact of local shock loads. Protective elements are also used as individual means of safety for people. Experience with the use of protective elements shows that the most effective are the multilayer elements. Under the influence of local shock loads, there is a high-rate elastic-plastic deformation of the material of the elements. Analysis of this process requires taking into account the dynamic properties of materials and finite deformations and displacements. It is shown that taking into account the dynamic properties of materials significantly affects the maximum dynamic stresses under impact loads [1-5]. Moreover, for equal loads and thicknesses of elements, the stresses and displacements differ substantially for different materials. Therefore, it is advisable to use multi-layer elements, different layers which consist of different materials. Analysis of the increase in the dynamic strength of protective elements is closely related to the dynamic properties of the material. An important feature of high-rate deformation of most structural materials is their hardening with increasing of strain rate. These properties can be reflected by means of the dependences of stress components on deformations and strain rates [6, 7]. For example, in a cylindrical coordinate system, these dependences have the form of

$$
\begin{array}{ll}
\sigma_{r}-\sigma=\frac{1}{\psi}\left(s_{r}-\frac{1}{z} z\right) & \tau_{r \varphi}=\frac{1}{\psi} \gamma_{r \varphi} \\
\sigma_{\varphi}-\sigma=\frac{1}{\psi}\left(s_{\varphi}-\frac{1}{z} z\right) & \tau_{\varphi z}=\frac{1}{\psi} \gamma_{\varphi z} \\
\sigma_{z}-\sigma=\frac{1}{\psi}\left(\varepsilon_{z}-\frac{1}{z} z\right) & \tau_{r z}=\frac{1}{\psi} \gamma_{r z}
\end{array}
$$

$\sigma=\frac{1}{3}\left(\sigma_{r}+\sigma_{\psi}+\sigma_{z}\right)$

$\varepsilon=\varepsilon_{r}+\varepsilon_{\varphi}+\varepsilon_{\varepsilon}$

where $\sigma_{r}, \nabla_{\varphi}, \sigma_{z}$ - components of normal stresses,

$\tau_{\gamma \psi}, \tau_{\gamma z}, \tau_{z \varphi}$ - components of tangential stresses,

$s_{Y}, s_{\omega}, z_{z}$-components of normal deformations,

$\gamma_{r \varphi}, \gamma_{r z}, \gamma_{z \varphi}$ - components of tangential deformations.

In the case of elastic deformations $\psi=\frac{1}{2 u}$ and dependences (2) are transferred to Hooke's law. In the case of plastic high-rate deformations $\psi=\frac{\pi}{2} \frac{z_{i}}{\sigma_{i}}$.

Dynamic hardening of materials reflects dependencies of intensities stresses $\sigma_{i}$ from strain intensity $\varepsilon_{i}$ and intensity of strain rate $\varepsilon_{i}$ : $\sigma_{i}=\sigma_{i}\left(\varepsilon_{i}, \varepsilon_{i}\right)$.

The dynamic characteristics of the material can be taken into account using known dependencies in the form of Perzyna [8]. 


$$
\sigma_{i}=\left[1+\left(\frac{a_{1}^{p}}{\gamma}\right)^{m}\right] E z_{i}
$$

where $E$ - elastic modulus;

$m$ and $r$ - coefficients of sensitivity to the rate deformation;

$\varepsilon^{p}$-rate of deformation in the plastic stage, or in the forme of Pierce [9]

$$
\sigma_{i}=\left[1+\left(\frac{\varepsilon_{1}^{p h}}{Y}\right)\right]^{3 n} E z_{i}
$$

The graphical representation of the dependence $\sigma_{i}=\sigma_{i}\left(\varepsilon_{i}, \dot{\varepsilon}_{i}\right)$., in contrast to [10] in this case, is supplemented with conditions that are used at different stages of the high-rate deformation of the material.

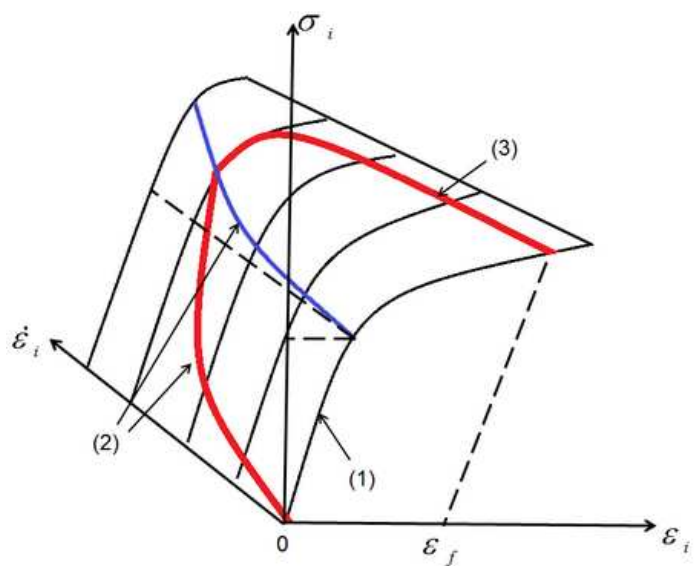

(1) $-\dot{\varepsilon}_{i}=0 ;(2)-0 \leq \dot{v}_{i}=a_{s}\left[1+\left(\frac{\varepsilon_{i}^{p h}}{\eta}\right)^{1 / n}\right] ;(3)-\Delta \varepsilon_{i} \leq 0$.

Fig. 1. Scheme of the stress intensity dependence from intensities strain and strain rate.

It is shown how the degree of hardening of the material varies with the rate of deformation and how the un-loading and the occurrence of residual stresses occur $\varepsilon_{f}$.

Numerical studies of the influence of impactor on a steel plate using the dependencies of Perzyna, Pierce and the elastic properties of the material are carried out. Fig. 2 shows the variation of the maximum equivalent stresses in time.

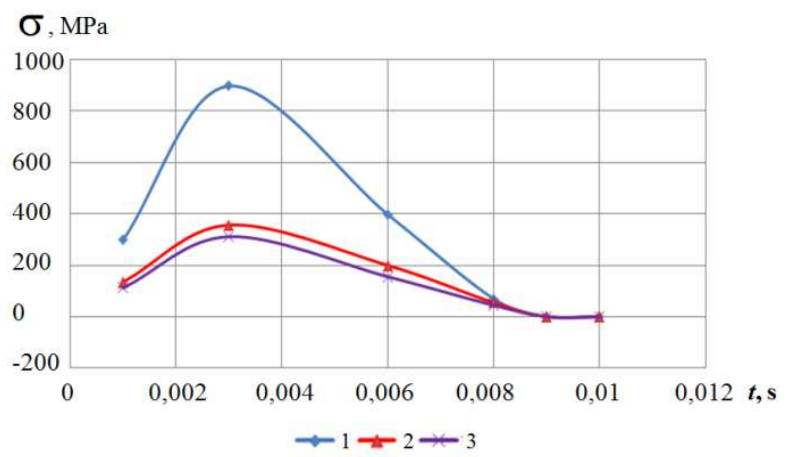

Fig. 2. Distribution of equivalent stresses in time, taking into account only the elastic properties of the material (1), the dependence of Perzyna (2) and Pierce's dependence (3).
Using the dependencies of Perzyna and Pierce gives close results. An attempt to solve the problem in an elastic formulation leads to significant errors. Equivalent stresses, when using the dependence of Perzyna, are somewhat higher than when using Pierce's dependence.

Using the dependence of Perzyna in the future leads to a margin of strength. The nature of the high-rate deformation of the material depends essentially on the mechanical properties and the parameters of hardening. Therefore Fig. 3 shows the variation of equivalent stresses in time in plates from different materials when the speed of impactor is $200 \mathrm{~m} / \mathrm{s}$.

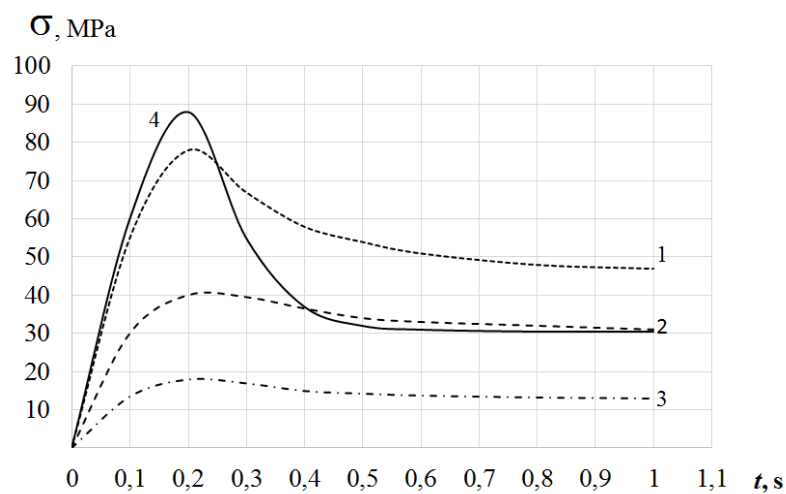

Fig. 3. Comparison of the distribution of the maximum equivalent stresses under the impact of a projectile at a speed of $200 \mathrm{~m} / \mathrm{s}$ in protective elements from various materials: 1 steel element; 2 - from aluminium, 3 - composite, 4 - titanium alloy.

It can be seen that higher tensile stresses develop for materials with higher degree of hardening. The values of displacement thus change in the reverse sequence. It follows that the elements should have the greatest protective properties if that combine materials with high hardness with materials that have high strain at lower stress levels.

\section{Description of the experiment and the results of numerical analysis}

As an example, the results of the action of a blade fragment ( $m=100 \mathrm{~g}$ ) under the influence of centrifugal force on the gas turbine engine corps element are being considered here. Fig. 4 shows a fragment of the corps of a gas turbine engine with a two-layer protective element and the distribution of displacements and stresses under the action of a blade fragment at a speed of $350 \mathrm{~m} / \mathrm{s}$. The thicknesses of the layers are $h_{1}=1.4 \mathrm{~mm}$ and $h_{2}=2 \mathrm{~mm}$ respectively.

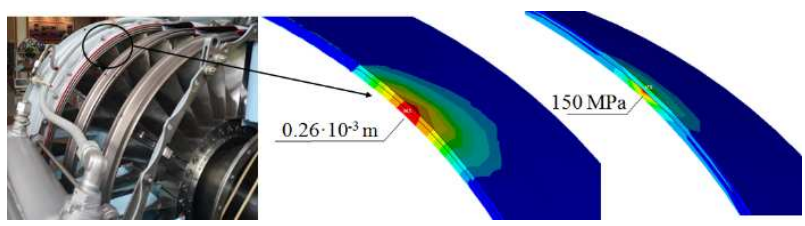

Fig. 4. Part of a gas turbine engine corps with a two-layer protective element, displacements and equivalent stresses in the element under the action of a blade fragment. 
When a similar fragment was applied to a singlelayer element with greater thickness $(h=8 \mathrm{~mm})$, the maximum stresses were $133 \mathrm{MPa}$. Thus, the two-layer element showed a much greater efficiency. Therefore, it is more appropriate to use multi-layered protective elements from different materials. Taking into account that titanium alloys tend to be more hardened than even steel ones, their capabilities should be used to perceive the main load in combination with materials capable of absorbing impact energy [11]. For example, we are going to consider a three-layer protective element of light armoured vehicles in Fig. 5.

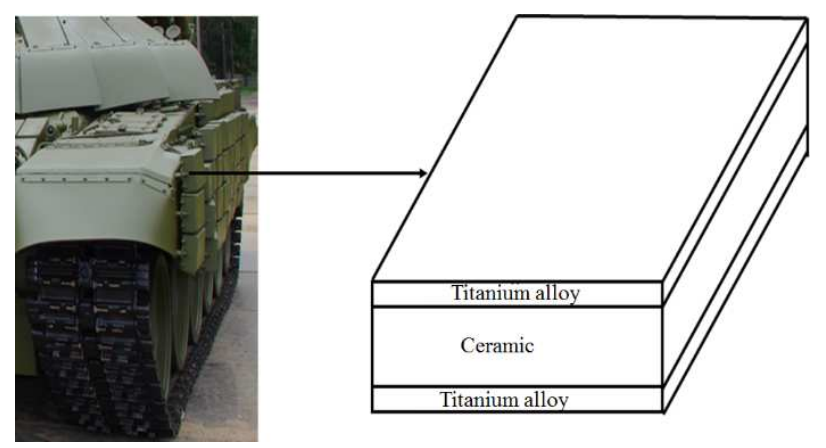

Fig. 5. Scheme of a three-layer protective element of light armoured vehicles.

The three-layer element of length $a=100 \mathrm{~mm}$ and width $b=100 \mathrm{~mm}$ is being considered here. The top and lower layers made of titanium alloy Ti6Al4V, have the same thickness $h_{1}=h_{3}=2 \mathrm{~mm}$. The thickness of the inner ceramic layer $h_{2}=3,2 \mathrm{~mm}$. The main mechanical properties of materials are following:

- for titanium alloy density $\rho=4420 \mathrm{~kg} / \mathrm{m}^{3}$, elastic modulus $E=1,19 \cdot 10^{11} \mathrm{~Pa}$, Poisson's ratio $v=0,342$, elastic limit $\sigma_{T}=9,1 \cdot 10^{8} \mathrm{~Pa}$,

- for ceramic: density $\rho=4900 \mathrm{~kg} / \mathrm{m}^{3}$; elastic modulus $E=6,32 \cdot 10^{11} \mathrm{~Pa}$; Poisson's ratio $v=0,204$,

- steel projectile of mass $m=0,1 \mathrm{~kg}: \rho=7800 \mathrm{~kg} / \mathrm{m}^{3}$, elastic modulus $E=2,06 \cdot 10^{11} \mathrm{~Pa}$, Poisson's ratio $v=0,3$, elastic limit $\sigma_{T}=2,99 \cdot 10^{8} \mathrm{~Pa}$, hardening module $E_{I}=$ $7,39 \cdot 10^{8} \mathrm{~Pa}$.

The impact of the projectile on a three-layer element at a speed of $600 \mathrm{~m} / \mathrm{s}$ is investigated. Fig. 6 shows the displacements and deformations, which occur in $3 \cdot 10^{-4} \mathrm{~s}$. In the top layer, the deformations have a clearly pronounced local character. In this case, a crater appears in the top layer, and an extensive region of deformations are in the average ceramic layer. But the middle layer prevents the further spread of large displacements into the interior of the element. However, the deformation region in the middle layer is extensive, which leads to absorption of the impact energy [12]. The lower layer limits the deformations of the middle layer.

Fig. 7 shows the isolines of equivalent stresses at the moment $t=0,3 \cdot 10^{-4} \mathrm{~s}$. The localization of high stresses in the crater area and the propagation of dynamic stresses in the middle layer are visible. In the third layer, much lower stresses are observed.

After the impact, the element has obvious damage in the form of a crater in the top layer, but since the new impact of the projectile on the protective element is most likely to occur outside the zone of the original local damage, the element practically retains its protective properties.

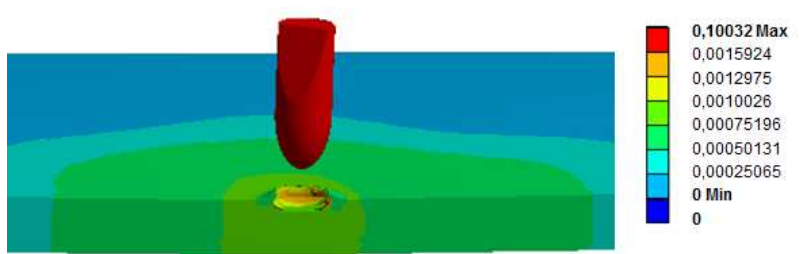

a)

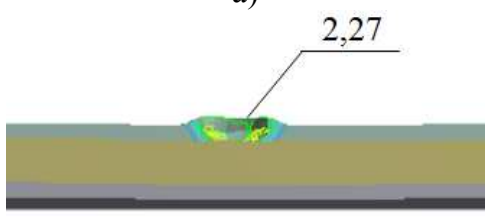

b)

Fig. 6. Displacements (unit: $\mathrm{m}$ ) (a) and deformations $\varepsilon^{p l}$ (unit: nondimensional quantity $\mathrm{m} / \mathrm{m}$ ) (b) in the three-layered protective element under the action of projectile.

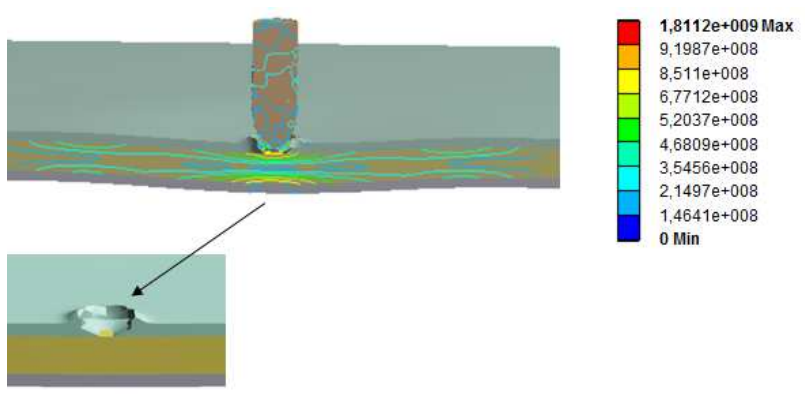

Fig. 7. Distribution of equivalent stresses (unit: $\mathrm{Pa}$ ) in a threelayer element at the speed impact of projectile of $600 \mathrm{~m} / \mathrm{s}$.

\section{Conclusions}

The results of numerical analysis show that the use of several layers makes the protective elements more effective than single-layer ones. Even with the use of two-layer elements from the same materials (like steel), which is typical of some elements of gas turbine corps (where there are elevated temperatures), these elements have higher protective properties than single-layer ones.

When layers of materials with high dynamic hardening characteristics are used in combination with a thicker ceramic layer, the efficiency of the protective structures is further improved. The top layer perceives the main shock load, when the projectile actions with high speeds. Due to hardening, plastic deformations occur in it and a crater is formed. In the middle layer, with a lower hardening, a considerable area of deformation arises.

Due to its size, the energy of its deformation is distributed throughout the volume and damped. The third layer limits the displacement of the middle layer. When the impactor is exposed on the top layer the local damage occurs, but it is limited by deformations of the middle layer. The whole element retains its protective properties when the projectile again hits the area unaffected by the first impact. In general, it can be noted 
that multi layered protection elements have increased strength properties and provide a reduction in the weight of the structure.

\section{References}

1. G. R. Johnson, W. H. Cook, in: Proceedings of 7th International Symposium on Ballistics, The Hague, The Netherlands, 541 (1983)

2. L. Kruszka, W. K. Nowacki, JTAM, 2, 259 (1996)

3. M. A. Meyers: Dynamics behavior of materials, (Wiley, New York, 1994)

4. V. T. Troshchenko, A, A, Lebedev, V. A. Strizhalo, Mechanical behaviour of materials under different types of loading (Logos, Kiev, 2000), in Russian

5. Y.S. Vorobiov, A. V. Kolodyazhny, V. I. Sevryukov, E. G. Yanyutin, High-speed deformation of structure elements (Naukova Dumka, Kiev, 1989), in Russian

6. Y. S. Vorobiov, L. Kruszka , N. Y. Ovcharova, Applied Mechanics and Materials, 566, 499 (2014)

7. Y. S. Vorobiov, L. Kruszka, N. Y. Ovcharova, Key Eng. Mater., 715, 216 (2016)

8. P. Perzyna, Thermodynamics of inelastic materials (PWN, Warsaw, 1978), in Polish

9. D. Peirce, D. R. J. Owen, A model for large deformations of elasto-viscoplastic solids at finite strains: computational issues, Finite Inelastic Deformations: Theory and applications (SpringerVerlag, Berlin, 1992)

10. Yu.S. Vorobiev, A.V. Kolodyazhny, M.V. Chernobryvko, L. Kruszka, in: Proceedings of the 15th International Scientific and Technical Conference on Environmental Engineering in the Maintenance of Military Complexes, Zakopane, Poland, 447 (2001), in Russian

11. Y. S. Vorobiov, N. Y. Ovcharova, L. Kruszka, in: Proceedings of the 5th International Conference on Nonlinear Dynamics ND-KhPI2016, Ukraine, 406 (2016)

12. Yu. S. Vorobiev, N. Yu. Ovcharova, Technical Mechanics, 3, 17 (2016), in Russian 